\title{
RESEARCH
}

Open Access

\section{The accuracy and safety of CT-guided iodine-125 seed implantation assisted by 3D non-coplanar template for retroperitoneal recurrent carcinoma}

Weijuan Jiang, Ping Jiang, Shuhua Wei, Yuliang Jiang, Zhe Ji, Haitao Sun, Jinghong Fan, Weiyan Li, Yuxia Shao and Junjie Wang ${ }^{*}$

\begin{abstract}
Purpose: To investigate the accuracy, dosimetric parameters, and safety of 3D-printing non-coplanar template (3DPNCT)-assisted CT guidance for radioactive iodine-125 (125I) seed implantation brachytherapy (RSI-BT) for retroperitoneal recurrent carcinomas

Methods and materials: We enrolled 15 patients with 17 retroperitoneal recurrent carcinomas after external beam radiotherapy (EBRT). All patients received CT-guided 125 I RSI-BT assisted by 3D-PNCT successfully. We compared the original needle insertion position, angular, and the needle tip distance deviations of preoperative plan with that of intraoperative in brachytherapy treatment planning system (B-TPS). The dosimetric parameters of RSI-BT were evaluated on preoperative plan, intraoperative real-time plan, and postoperative plan, including D90, D100 (the dose to $90 \%$ and $100 \%$ of the target volume), V100, V150, and V200 (the volume receives 100\%, 150\%, and 200\% of the prescribed doses). The quality assurance of RSI-BT evaluated on conformal index (Cl), external index (EI), and homogeneity index $(\mathrm{HI})$ of the targets were compared among preoperative plan, intraoperative real-time plan, and postoperative plan. The perioperation complications and RSI-BT-related toxicity were assessed.

(Continued on next page)
\end{abstract}

\footnotetext{
* Correspondence: junjiewang_edu@sina.cn

Department of Radiation Oncology, Peking University Third Hospital, No.49 Huayuan North Road, Haidian District, Beijing 100191, People's Republic of China
}

(c) The Author(s). 2020 Open Access This article is licensed under a Creative Commons Attribution 4.0 International License, which permits use, sharing, adaptation, distribution and reproduction in any medium or format, as long as you give appropriate credit to the original author(s) and the source, provide a link to the Creative Commons licence, and indicate if changes were made. The images or other third party material in this article are included in the article's Creative Commons licence, unless indicated otherwise in a credit line to the material. If material is not included in the article's Creative Commons licence and your intended use is not permitted by statutory regulation or exceeds the permitted use, you will need to obtain permission directly from the copyright holder. To view a copy of this licence, visit http://creativecommons.org/licenses/by/4.0/. The Creative Commons Public Domain Dedication waiver (http://creativecommons.org/publicdomain/zero/1.0/) applies to the data made available in this article, unless otherwise stated in a credit line to the data. 


\begin{abstract}
(Continued from previous page)
Results: The median follow-up was 8.2 months (range 1-18.5 months). One patient was lost to follow-up after RSIBT. Fourteen patients were assessed for response rate and toxicity. The mean entrance point distance deviation for all 165 needles was $4.50 \pm 4.10 \mathrm{~mm}$ (range, $0-30$ ). The mean angular deviation was $2.70 \pm 3.00^{\circ}$ (range, $0-20$ ). The needle tip distance deviation was $6.90 \pm 6.00 \mathrm{~mm}$ (range, - 30-28). D90 for preoperative plan, intraoperative plan, and postoperative plan were $140.55 \pm 23.93,124.25 \pm 28.04$, and $128.98 \pm 22.75$, respectively. There was significant difference between $\mathrm{D} 90$ of preoperative plan with that of intraoperative plan $(p=0.036)$. Four lesions reached $C R$, six lesions reached PR, three lesions were SD, and three lesions were PD. Four patients with moderate pain became mild, and two with mild pain relieved completely after RSI-BT. The other parameters showed no differences among preoperative plan, intraoperative plan, and postoperative plan. The perioperative complications were observed in four patients, including three patients of grade 1 and one patient of grade 2 . No $\geq$ grade 3 side effects were observed.
\end{abstract}

Conclusion: CT-guided 125I RSI-BT assisted by 3D-PNCT was a safe, accurate, and feasible strategy for recurrent carcinomas located in the retroperitoneal regions.

Keywords: LDR brachytherapy, Seed implantation, 3D-printing non-coplanar template, Retroperitoneal carcinoma, Accuracy

\section{Introduction}

The anatomic construction in retroperitoneal region was deep and complex due to a majority of important organs located there, such as the blood vessels and spinal cord. The most common carcinomas in retroperitoneal regions were metastasis originated from cervical cancer, pancreatic cancer, gastric cancer, and adrenal metastasis, while the primary carcinoma was mainly soft tissue sarcoma [1-5]. The standard treatment approach was EBRT plus chemotherapy for recurrent carcinoma after surgery or metastasis in the retroperitoneal region. However, the majority of recurrent patients after EBRT or EBRT combined with chemotherapy were often resistant to re-radiation or chemotherapy [6-10]. The salvage approach for recurrent patients was always deemed as a challenge, most of which was palliative treatment and mainly for relieve of the symptoms. The response rate (RR) of chemotherapy, which is the second line or salvage treatment regimen for cervical, pancreatic, and gastric carcinoma, was unfavorable [11-13]. Most patients suffered from severe pain and suboptimal quality of life (QOL).

125I RSI-BT belongs to low-dose rate brachytherapy (LDR-BT) which delivers locally high doses inside the targets and rapidly drops off surrounding the normal tissues [14]. Transperineally ultrasound (US)-guided 125I RSI-BT was a standard modality for prostate cancer because of the excellent outcomes and minimally invasive procedure compared with surgery or EBRT $[15,16]$. The disadvantages of US-guided RSI-BT included (1) twodimensional image reconstruction and low resolution; (2) unsuitable for carcinomas located in the head and neck, thoracic, retroperitoneal, pelvic, and spinal cord due to air or bony construction interferences; (3) the available commercial brachytherapy treatment planning system (B-TPS) was special for prostate carcinoma, with all needle arrangement kept in parallel and high dependence on ultrasound guidance; (4) the available template was rigid and only for parallel needle trajectory, which was unsuitable for non-coplanar insertion. The suboptimal dose conformity for special location tumors was unavoidable. With the development of 3D-printing techniques, the individualized template was invented and named as 3D-printing non-coplanar template (3DPNCT) [17]. However, 3D-PNCT combined with CT guidance for long distance puncture pathway achieved optimal dosimetry conformality in the targets. Thus, the accuracy and safety of 3D-PNCT-assisted CT guidance RSI-BT were investigated in this study.

\section{Methods and materials}

\section{Patient indication selection}

Totally, 15 patients with 17 lesions from recurrent carcinomas located in retroperitoneal regions who received EBRT were enrolled in this study from August 2016 to September 2019 (Table 1). The pre-RT doses ranged from 30 to $80 \mathrm{~Gy}$. The median tumor volume was 23.1 cc (range, 5.1-68.6 cc). The indications for selected patients in this study were as follows: (1) pathological or radiological diagnosis was confirmed; (2) the diameter $\leq$ $5 \mathrm{~cm}$ and no invasion to intestinal tube or spine; (3) preoperative plan showed that the needle channel pathway and the prescribed doses were satisfactory; (4) predicted survival time $\geq 3$ months. The exclusion criteria were as follows: (1) severe coagulation functional disorder, (2) tumor invasion into the spine or intestine, (3) and preoperative plan showed that there was no satisfied needle puncture pathway. The study was approved by the ethics committee of our hospital. 
Table 1 General information

\begin{tabular}{|c|c|c|}
\hline & Number & Percentage (\%) \\
\hline \multicolumn{3}{|l|}{ Gender } \\
\hline Male & 7 & 46.7 \\
\hline Female & 8 & 53.3 \\
\hline \multicolumn{3}{|l|}{ Age, years } \\
\hline Median (range) & $58(38-78)$ & \\
\hline \multicolumn{3}{|l|}{ Primary tumor location } \\
\hline Esophagus & 3 & 20 \\
\hline Pancreas & 1 & 6.7 \\
\hline Cervix & 4 & 26.7 \\
\hline Corpus uteri & 3 & 20 \\
\hline Liver & 1 & 6.7 \\
\hline Colon & 1 & 6.7 \\
\hline Ureter & 1 & 6.7 \\
\hline Cardia & 1 & 6.7 \\
\hline \multicolumn{3}{|l|}{ Pathological type } \\
\hline Squamous cell carcinoma & 7 & 46.7 \\
\hline Adenocarcinoma & 2 & 13.3 \\
\hline Hepatocellular carcinoma & 1 & 6.7 \\
\hline Urothelium carcinoma & 1 & 6.7 \\
\hline Endometrioid adenocarcinoma & 3 & 20 \\
\hline Neuroendocrine carcinoma & 1 & 6.7 \\
\hline \multicolumn{3}{|l|}{ Previous treatment } \\
\hline EBRT & 15 & \\
\hline Prescribed doses (median, range) & $50.4(30-80)$ & \\
\hline \multicolumn{3}{|l|}{ Implantation location } \\
\hline Retroperitoneal lymph node & 13 & 76.5 \\
\hline Adrenal gland & 3 & 17.6 \\
\hline Pancreas & 1 & 5.9 \\
\hline
\end{tabular}

\section{Patient preparation and preoperative plan}

Patients were set-up on the CT simulator and immobilized at prone position with vacuum mattress. Both native and contrast CT scan were performed with 5$\mathrm{mm}$ thickness before RSI-BT. The CT scan slices were transferred into B-TPS (Beijing University of Aeronautics and Astronautics and Beijing Astro Technology Co., Ltd) for preoperative plan. Planning system source data originated from the latest official manuscripts of the American Association of Physicists in Medicine (AAPM) [18, 19].

\section{The principle of preoperative plan design}

The needles should be kept in parallel, with distance of $1-1.5 \mathrm{~cm}$. If the organs at risk (OARs) are interference for the needles, non-coplanar needle distribution was adapted to satisfy the target dose conformity and the lowest doses to normal tissue. Then, we delineated targets and OARs and defined prescribed doses and limitation of OARs. Clinical target volume (CTV) was expanded from gross tumor volume (GTV) by $5-6 \mathrm{~mm}$ in three dimensions. Prescribed doses were 110-160 Gy; the radioactivity of $125 \mathrm{I}$ seed was $0.4-0.7 \mathrm{mCi}$ (the seed model was 6711-1985, Shanghai GMS Pharmaceutical Co., Ltd). The principles of seed distribution were sparse in the center and dense at the peripheral zone of the targets (Fig. 1).

\section{D-PNCT design and production}

The B-TPS data were imported into 3D imaging and reverse engineering software for individualized digital modeling design. 3D-PNCT was obtained by a 3D curing rapid prototyping machine and the material processing of medical curing resins. The 3D-PNCT contained certain information such as body surface characteristics, $\mathrm{X}$ and Y-axis coordinates, 2-3 stable needles, and dummy needle channel holes.

We classified recurrent retroperitoneal carcinoma into 3 subgroups according to the recurrent locations referenced to the spinal cord. The definition of subgroup included the following: (1) type 1, the recurrent tumors located in the left side of the spinal cord and the front margin did not spread to the middle line, the needles are inserted from the left side; (2) type 2, the recurrent tumor is located before the front edges of the spinal cord, the needles are inserted from two sides; (3) type 3, the recurrent tumors located in the right side of the spinal cord and the front margin did not spread to the middle line of the spinal cord, the needles are inserted from the right side (Fig. 2).

\section{The work-flow of $125 \mathrm{I}$ RSI-BT}

The procedures of 125I RSI-BT were as follows: (1) patients were set-up again with template fixed on the patient body by 2-3 stable needles; (2) CT-scan was performed to verify the stable needles position. If the deflection errors were $>2 \mathrm{~mm}$, we adjusted the needles' position until the deviation $\leq 2 \mathrm{~mm}$. (3) The other implant needles were inserted into the targets; (4) CT scan was performed again to check the tips of the needles' position. If the deflection errors were $>2 \mathrm{~mm}$, we made a fine adjustment until the errors were $\leq 2 \mathrm{~mm}$; (4) $125 \mathrm{I}$ seeds were implanted with applicators according to the preoperative plan and the needles withdraw to the skin below $1 \mathrm{~cm}$; (5) CT scans were conducted again to verify the seed distribution. If the seed distribution did not meet the requirements of preoperative plan, seed savage implantation was performed immediately, and all the needles were taken out; (6) CT scan was performed again and transferred the slices into the B-TPS for postoperative dose parameter calculation [17]. After RSI-BT, 

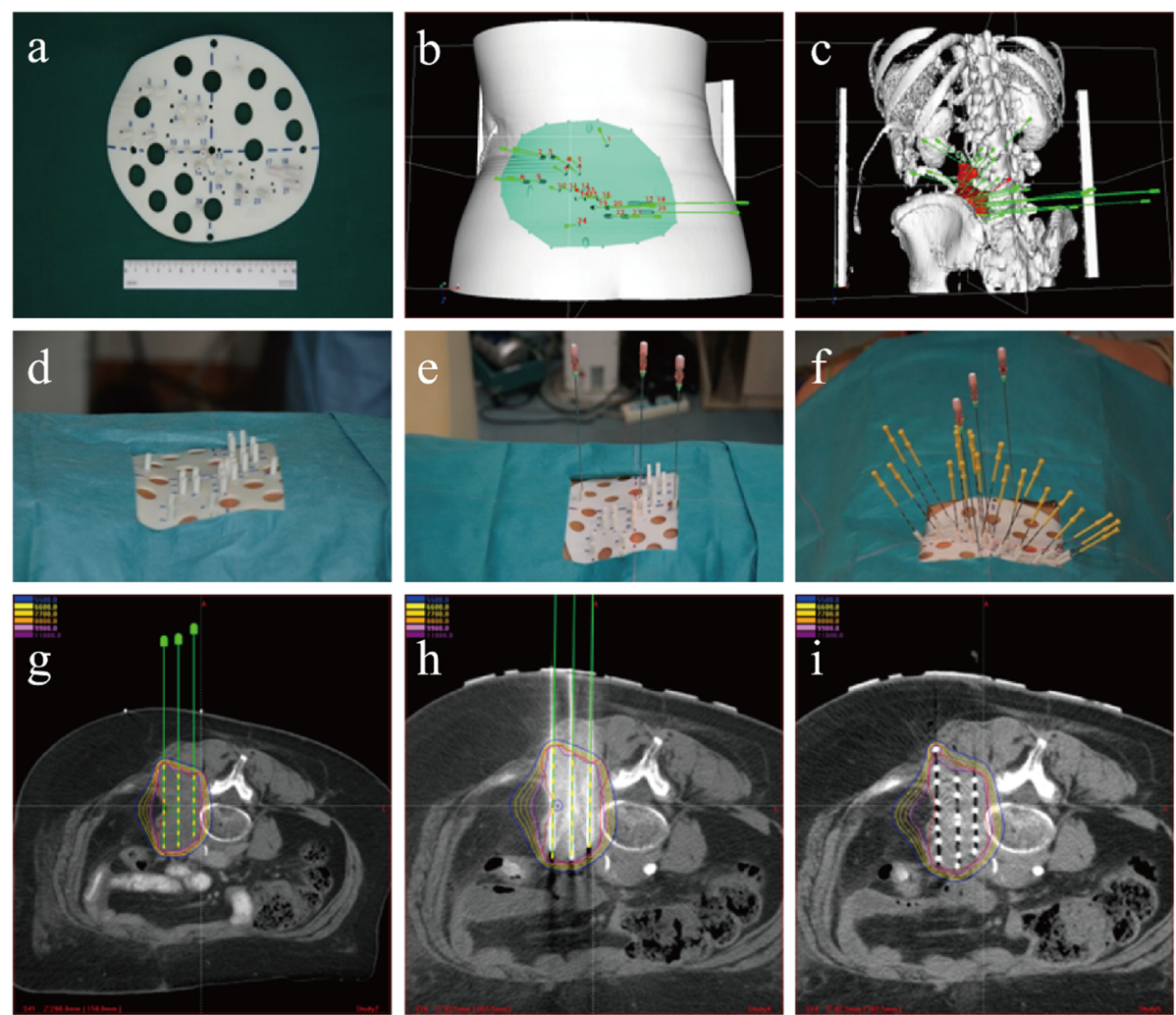

Fig. 1 The work-flow of 3D-PNCT-assisted CT guidance RSI-BT. a 3D-PNCT. b 3D-PNCT reconstruction on CT images. c 3D model view of bony anatomy and the puncture needle distribution reconstruction. $\mathbf{d}$ 3D-PNCT set-up: e stable needle insertion based on preplan, $\mathbf{f}$ seed needle insertion, $\mathbf{g}$ preplan, $\mathbf{h}$ intraoperative real-time plan, and $\mathbf{i}$ postoperative plan

the patients returned to patient wards and received perioperative prophylactic antibiotics and hemostasis for 1 day and discharged $24 \mathrm{~h}$ later.

\section{Postoperative plan for dosimetric verification}

The D90, D100, V100, V150, and V200 were recognized as the dosimetric parameters of tumor target. The quality assurance comparison of RSI-BT involved CI, EI, and $\mathrm{HI}$ among preoperative plan, real-time plan, and postoperative plan.

\section{Definition of end points}

\section{Main end points}

(1) To evaluate the accuracy of needle distributions, we compared the preoperative plan with intraoperative on B-TPS. The CT images in the preoperative plan were fused with intraoperative real-time CT scan images depending on bone construction as the references. The needle tip positions, the needle angles, and the tip depth were measured. (2) The D90 (doses delivered to $90 \%$ of the target volume), D100, V100 (the percentage of the target volume receiving at least $100 \%$ of the prescribed

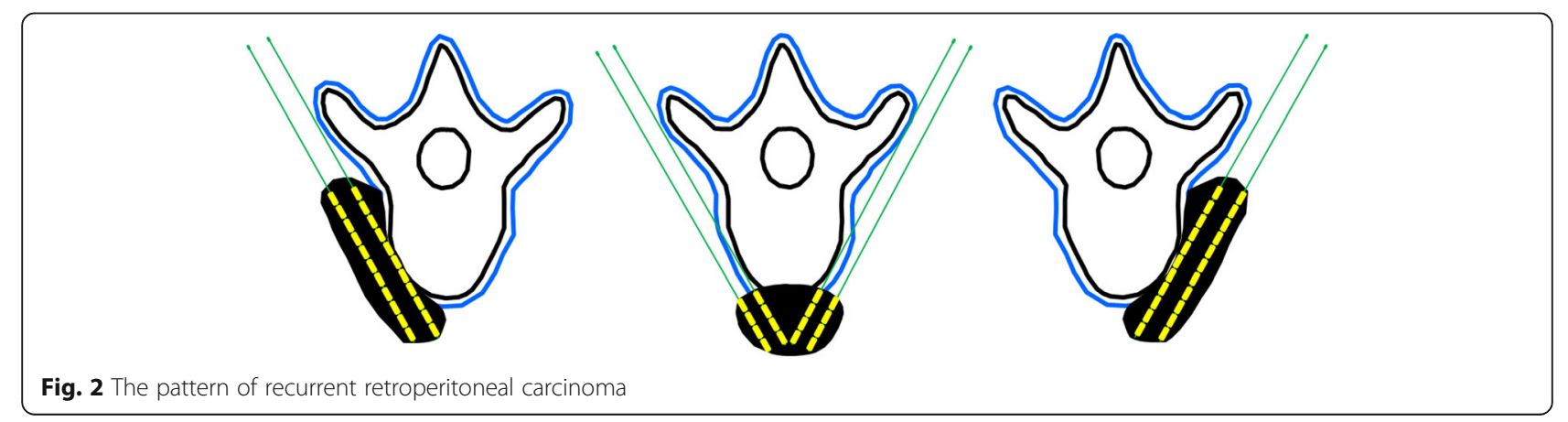


doses), V150, and V200 were calculated. (3) The quality of RSI-BT in the preoperative, real-time, and postoperative plan targets were compared with CI, HI, and EI.

\section{Secondary main point}

(1) Perioperation side effect assessment included bleeding, fever, infection, fistula; (2) the radiation-related complications were assessed according to RTOG Common Toxicity Criteria; and (3) pain relief rate was evaluated.

\section{Follow-up}

After RSI-BT, routine follow-up was performed every 3 months in the first 2 years and every 6 months from 3 to 5 years, followed by annual evaluation. CT scans of the thorax and abdomen were part of the follow-up for contrast. Patients underwent clinical evaluation and laboratory testing. The evaluation of efficacy was based on the Response Evaluation Criteria in Solid Tumors (RECIST) v1.1, including complete response (CR), partial response $(\mathrm{PR})$, progressive disease $(\mathrm{PD})$, and stable disease $(\mathrm{SD})$. Adverse reactions were evaluated by the Common Terminology Criteria for Adverse Events (CTCAE) v 4.0 (CTCAE 2010) [20, 21]. Pain was assessed using a numerical rating scale (NRS) which was categorized into five grades: 0 for no pain, 1-3 for mild pain, 4-6 for moderate pain, 7-9 for severe pain, and 10 for unbearable pain. The pain score in 1 month after the treatment was compared with that of pre-operation.

\section{Statistical analysis}

The characteristics of patients were expressed as continuous variables and/or categorical variables. Continuous variables were compared using the $t$ test or ranksum test, whereas the categorical variables were compared using the chi-square or Fisher's exact test. ORR was expressed based on the number and percentage of patients. The SPSS 21.0 software (SPSS, Chicago, IL) was used for statistical analysis. The $P$ value $<0.05$ was considered as statistical significance.

\section{Results}

The median follow-up time was 8.2 months (range, 118.5 months). One patient was lost to follow-up. The 15 patients with 17 lesions were enrolled for accurate assessment, and 14 patients were involved for side effect analysis. The median GTV in the preoperative plan, intraoperative plan, and postoperative plan were $26.41 \pm$ $16.2,26.71 \pm 17.43$, and $26.72 \pm 17.46$, respectively $(p>$ $0.5)$. The mean needle tip deviation for all 165 needles was $4.50 \pm 4.10 \mathrm{~mm}$ (range, $0-30, p<0.001$ ). The mean angular deviation was $2.70 \pm 3.00^{\circ}$ (range, $0-20, p<$ $0.001)$. The needle depth deviation was $6.90 \pm 6.00 \mathrm{~mm}$ (range, $-30-28, p<0.001$ ) (Table 2). D90, D100, V100,
V150, and V200 for preoperative plan, intra-operative plan, and postoperative plan were $140.55 \pm 23.93,124.25$ $\pm 28.04,128.98 \pm 22.75 ; 66.70 \pm 16.77,58.14 \pm 21.24$, $61.51 \pm 15.86 ; 24.83 \pm 15.94,22.71 \pm 12.10,23.64 \pm$ $13.75 ; 20.02 \pm 13.32,17.45 \pm 10.15,18.74 \pm 11.53$; and $14.51 \pm 11.02,12.38 \pm 8.07,23.59 \pm 8.97$, respectively. The CI, EI, and HI were 0.54 $\pm 0.16,0.50 \pm 0.15,0.50 \pm$ $0.17 ; 0.90 \pm 1.00,0.91 \pm 0.90,1.06 \pm 0.99$; and $0.22 \pm$ $0.07,0.25 \pm 0.09,0.21 \pm 0.08$, respectively (Table 3 ). There were no statistically significant differences in D100, V100, V150, V200, CI, EI, and HI in GTV between preplan and intraoperative plan, preoperative plan and intraoperative plan, and intraoperative plan with that of postoperative plan $(p>0.05)$, while there was only significant differences between D90 $(p<0.05)$ of preoperative plan with that of intraoperative plan. There were no differences between intraoperative dose optimization with that of postoperative plan.

When it comes to response rate at 3 months after seed implantation, CR was observed in four lesions. PR was shown in six lesions. SD was observed in three lesions. PD was observed in three lesions. Among them, four patients with moderate pain became mild; two with mild pain were relived completely after RSI-BT. One patient had pain again 4 months later, and the other five patients had pain control until the last follow-up or death. The median time to achieve pain control was 8 months (range, 4-13 months). The perioperation complications were observed in four patients, including three of grade 1 and one of grade 2 . No $\geq$ grade 3 side effects were observed (Table 4).

\section{Discussion}

High-dose-rate BT (HDR-BT) is a very important modality in RT, and commonly used in breast cancer, cervical cancer, prostate cancer, and skin cancer treatment [22, 23]. The outcome of HDR-BT combined with surgery for selected recurrent soft tissue carcinomas were favorable, however, with the complication rates ranged from 15 to $50 \%$ [24]. The 125I RSI-BT belongs to LDRBT and has advantages as follows: (1) single performance; (2) real-time image guidance; (3) the local boost dose to target; (4) minimal invasion and fast recovery.

In order to expand the indications of RSI-BT, CT guidance technique was integrated into RSI-BT in 2002 in China, which was used to treat head and neck, thoracic, abdomen, and spinal vertebrate carcinoma [25-30]. The CT-guided 125I RSI-BT was a very safe and effective approach for anti-cancer treatment, especially for recurrent or metastatic carcinomas. The majority of recurrent carcinomas located in retroperitoneal regions had lost the opportunity to further resection or reradiation after EBRT due to the surrounding OARs' dose limitation such as the intestine, spinal cord, and blood 
Table 2 The deviation of position, angle, and distance of needles between preoperative plan and intraoperation plan in B-TPS

\begin{tabular}{|c|c|c|c|c|c|c|c|c|c|}
\hline \multirow{2}{*}{$\begin{array}{l}\text { No. of } \\
\text { needles }\end{array}$} & \multicolumn{3}{|c|}{ Deviation of position $(\mathrm{mm})$} & \multicolumn{3}{|c|}{ Deviation of angle $\left({ }^{\circ}\right)$} & \multicolumn{3}{|c|}{ Deviation of distance $(\mathrm{mm})$} \\
\hline & Mean & Range & SD & Mean & Range & $\overline{S D}$ & Mean & Range & $\overline{S D}$ \\
\hline 165 & $4.5(0.000)$ & $0-30$ & 4.1 & $2.7(0.000)$ & $0-20$ & 3.0 & $6.9(0.000)$ & $-30 \sim 28.1$ & 6.0 \\
\hline
\end{tabular}

vessels. Yao et al. reported that 17 patients with 19 retroperitoneal lymph node recurrences after EBRT underwent CT-guided 125I RSI-BT. The actuarial D90 of postoperative plan was 100-198 Gy (median, 126.5 Gy). Nine patients with pain decreased to mild pain 1-3 weeks after RSI-BT. Pain-free survival time ranged 2-15 months (median, 5 months). The overall survival (OS) rate was $100 \%$. The median local control (LC) time was 15 months. The 1 - and 2 -year LC rate was $63.2 \%$ and $42.1 \%$, respectively. Twelve patients $(70.6 \%)$ died of distant metastasis. Two patients (11.8\%) survived with distant metastases and without evidence of local recurrence. Median OS time was 10 months. The 1- and 2year OS rate were $38.1 \%$ and $15.3 \%$, respectively. No major complications related to RSI-BT occurred during or after treatment [31]. There were some drawbacks as follows: (1) It is a long period to train a skilled puncture personnel; (2) the quality control of RSI-BT was not often concomitant with preoperative plan design due to OAR interferences such as blood vessels, nerve, and bone construction. The analysis of CT-based dosimetry revealed that dose coverage of RSI-BT postoperative plan was often lower than that in preoperative plans; (3) the operation time average was $2-3 \mathrm{~h}$ because $\mathrm{CT}$ scan was conducted to check every needle position for several times. Thus, investigators explored the possibility of template-assisted CT guidance to overcome these limitations, which theorized the precision placement of seeds to the periphery of the targets in a highly conformal manner, ensured good tumor control, and minimized the doses to the surrounding OAR.

With the development of 3D-printing techniques, the 3D-printing substance was explored across the medical field. Gross et al. used stereolithography printers to print the temporal bone anatomy of patients with congenital aural atresia to plan atresia plasticity [32]. D'Urso et al. published their early study with 3D-printed models of craniofacial and maxillofacial defects in 45 patients [33]. Their experience showed improved measurement accuracy and suggested modest improvement in operative time while improving patient education [34]. BT plays an important role in cancer treatment as monotherapy or combined with EBRT. The key techniques were the image guidance combined with template assistance which assured the applicators or needles placed into the targets accurately as preoperative plan designed, especially for the breast, prostate, and skin cancer [14, 15]. Those cancer anatomic locations were relatively simple, and the applicators or needles can easily be inserted into the interested targets and kept in parallel arrangements. The optimal dose patterns of the targets were achieved to maximize the doses to the cancer and minimize the doses to normal tissues. However, the carcinomas located in the head and neck, thoracic, retroperitoneal, pelvic, and spinal cord sites were very different from the above location. The rigid template for prostate or cervical cancer cannot satisfy the requirements for complex anatomy construction and shape of tumors with RSI-BT.

Table 3 The dosimetric parameters of 1251 seeds implantation $(x \pm s)$

\begin{tabular}{|c|c|c|c|c|c|c|}
\hline & Preoperative & Intraoperative & Postoperative & $P^{*}$ & $P^{* *}$ & $P^{* * *}$ \\
\hline Number of needles & $10.76 \pm 4.05$ & $9.76 \pm 3.80$ & $9.82 \pm 3.75$ & 0.010 & 0.007 & 0.332 \\
\hline Number of seeds & $48.00 \pm 16.80$ & $46.82 \pm 18.25$ & $47.88 \pm 18.75$ & 0.595 & 0.962 & 0.070 \\
\hline GTV volume (cm3) & $26.41 \pm 16.2$ & $26.71 \pm 17.43$ & $26.72 \pm 17.46$ & 0.585 & 0.629 & 0.952 \\
\hline D90 & $140.55 \pm 23.93$ & $124.25 \pm 28.04$ & $128.98 \pm 22.75$ & 0.036 & 0.102 & 0.338 \\
\hline D100 & $66.70 \pm 16.77$ & $58.14 \pm 21.24$ & $61.51 \pm 15.86$ & 0.169 & 0.459 & 0.500 \\
\hline V100 & $24.83 \pm 15.94$ & $22.71 \pm 12.1$ & $23.64 \pm 13.75$ & 0.142 & 0.317 & 0.129 \\
\hline V150 & $20.02 \pm 13.32$ & $17.45 \pm 10.15$ & $18.74 \pm 11.53$ & 0.058 & 0.302 & 0.056 \\
\hline V200 & $14.51 \pm 11.02$ & $12.38 \pm 8.07$ & $23.59 \pm 8.97$ & 0.119 & 0.451 & 0.072 \\
\hline $\mathrm{Cl}$ & $0.54 \pm 0.16$ & $0.50 \pm 0.15$ & $0.50 \pm 0.17$ & 0.68 & 0.342 & 0.985 \\
\hline $\mathrm{El}$ & $0.90 \pm 1.00$ & $0.91 \pm 0.90$ & $1.06 \pm 0.99$ & 0.981 & 0.553 & 0.119 \\
\hline $\mathrm{HI}$ & $0.22 \pm 0.07$ & $0.25 \pm 0.09$ & $0.21 \pm 0.08$ & 0.380 & 0.637 & 0.117 \\
\hline
\end{tabular}

*Refers to preoperative vs. intraoperative

${ }^{*}$ Refers to preoperative vs. postoperative

***Refers to intraoperative vs. postoperative 
Table 4 Perioperative and postoperative complication of seeds implantation

\begin{tabular}{|c|c|c|c|c|c|}
\hline & & \multicolumn{4}{|c|}{ RTOG/CTC scoring schema } \\
\hline & & $\overline{\mathrm{G} 1}$ & G2 & G3 & G4 \\
\hline \multirow[t]{5}{*}{ Perioperative } & Pneumothorax & 0 & 1 & 0 & 0 \\
\hline & Hematemesis & 1 & 0 & 0 & 0 \\
\hline & Hemorrhage of digestive tract & 1 & 0 & 0 & 0 \\
\hline & Fever & 0 & 0 & 0 & 0 \\
\hline & Infection & 0 & 0 & 0 & 0 \\
\hline Postoperative & Skin & 1 & 0 & 0 & 0 \\
\hline
\end{tabular}

Huang et al. first reported the accuracy of RSI-BT in 31 patients with recurrent and locally advanced head and neck malignant tumors. The preliminary study confirmed that this approach was easier and more accurate. The D90, V100, and V150 all meet the treatment requirements [35]. The carcinomas that occurred in the head and neck regions were relatively stable and superficial, so it is relatively easy for template to fix with the patient anatomic construction, and the needle puncture deviation was infrequent. We applied the individualized template for the carcinomas in a deep location and longdistance puncture pathway. The body 3D-printing individual, digital, and coordinate template was invented in 2015. The 3D-PT was classified into 3D-printing coplanar template (3D-PCT) and 3D-PNCT. The indications of 3D-PCT were only for needle insertion kept in parallel way, and the needle hole interval was $0.5-10 \mathrm{~mm}$ which assured that the target dose pattern meets the preoperative plan requirements. When the needle pathway was impeded by OARs with 3D-PCT assistance, the needles were adjusted to the near holes to keep away from OARs or established an artificial channel with puncher for bone construction. The indications of 3DPNCT can apply to almost all the carcinomas in different locations for RSI-BT; the target dose conformality of which was optimal [36]. The advantages of 3D-PNCT included the following: (1) The X-and Y-axis coordinate located in the center of the template which can match with the marked lines on the patient body surface; (2) extra 2-3 stable needle holes designed on the template was used to immobilize the template on patient body. The stable needle position should be designed near the bony construction as references and easy to compare with preoperative plan images; (3) the dummy needle channels were designed to overcome the organ motion during operation. Salvage seed implantation can be performed immediately due to suboptimal dose pattern on the real-time plan optimization, in order to avoid the patients returning to hospital for second time seed implantation. The increasing accuracy and universality of RSI-BT promoted it to be an alternative minimally invasive and precise ablation approach which was deemed as stereotactic ablation brachytherapy (SABT).

The effective treatment modality for recurrent carcinomas in retroperitoneal locations still faces a giant challenge, especially for recurrence after EBRT. Our preliminary study showed the possibility and safety of 3D-PNCT-assisted CT-guided RSI-BT, which suggested that intra-operative D90 was significantly different with that in preoperative plan, and there was no significant difference between preoperative plan and postoperative plan through real-time dose optimization. The needles' position deviation for recurrent carcinomas located in retroperitoneal region might occur, due to the long distances puncture, the organ motion, and OAR interferences. The difference of needles' tip position, angles, and depth among preoperative plan, intraoperative plan, and postoperative plan were all significant, but no significant differences were seen on the CI, EI, and HI among preoperative plan, real-time plan, postoperative plan, which suggested that the intraoperative needle position adjustment and dose optimization were very important. The postoperative plan D90 of RSI-BT would reach the preoperative plan designed requirements even for carcinomas located in depth position. The perioperation complications were moderate. The radiation-related toxicity rates were acceptable.

\section{Conclusion}

3D-PNCT-assisted CT-guided RSI-BT is a safe and effective approach in treatment of recurrent carcinomas in retroperitoneal regions. It is worth warranted to conduct more multiple, prospective, and randomized clinical trials to compare the long-term efficacy of 125I RSI-BT with second line treatment.

\section{Acknowledgements}

We all thank the funding support from the National Natural Science Foundation of China.

\section{Authors' contributions}

Dr. Weijuan Jiang collected the data, drafted the manuscript, and did the statistical analysis. Dr. Ping Jiang, Dr. Yuliang Jiang, and Dr. Zhe Ji were in charge of verifying patients' treatment plans and directing manuscript 
writing. Dr. Shuhua Wei collected the material and reviewed and corrected this manuscript. Haitao Sun, Jinghong Fan, Weiyan Li, and Yuxia Shao were responsible for collection of clinical data. Dr. Junjie Wang was responsible for the supplementation/refinement of clinical data and carried out statistical analyses. The authors read and approved the final manuscript.

\section{Funding}

This work was supported by the National Natural Science Foundation of China (61631001 to J. Wang).

\section{Availability of data and materials}

No additional data are available.

\section{Ethics approval and consent to participate}

This study was approved by the Ethics Committee of Peking University Third Hospital. All patients or their representatives provided written informed consent for inclusion of patients in the study.

\section{Consent for publication}

Not applicable

\section{Competing interests}

All authors declared that there are no conflicts of interest.

Received: 14 May 2020 Accepted: 16 November 2020

Published online: 25 November 2020

\section{References}

1. Lewis JJ, Leung D, Woodruff JM, et al. Retroperitoneal soft-tissue sarcoma. Analysis of 500 patients treated and followed at a single institution. Ann Surg. 1998;228:355-65.

2. Demetri GD, Antonia S, Benjamin RS, et al. Soft tissue sarcoma. J Natl Compr Cancer Network. 2010:8:630-74.

3. Gronchi A, Strauss DC, Miceli R, et al. Variability in patterns of recurrence after resection of primary retroperitoneal sarcoma (RPS): a report on 1007 patients from the multi-institutional collaborative transatlantic RPS working group. Ann Surg. 2016:263:1002-9.

4. von Mehren M, Randall RL, Benjamin RS, et al. National Comprehensive Cancer Network. Soft tissue sarcoma, version 2. 2014. JNatlCompr Canc Netw. 2014;12:473-83.

5. Singer S, Corson JM, Demetri GD, et al. Prognostic factors predictive of survival for truncal and retroperitoneal soft-tissue sarcoma. Ann Surg. 1995 221:185-95

6. Ohri N, Garg MK, Aparo S, et al. Who benefits from adjuvant radiation therapy for gastric cancer? A meta-analysis. Int J Radiat Oncol Biol Phys. 2013:86:330-5.

7. Strander H, Turesson I, Cavallin-Stahl E. A systematic overview of radiation therapy effects in soft tissue sarcomas. Acta Oncol. 2003:42:516-31.

8. Bates JE, Dhakal S, Mazloom A, Constine LS. The benefit of adjuvant radiotherapy in high-grade nonmetastatic retroperitoneal soft tissue sarcoma: a SEER analysis. Am J Clin Oncol. 2018;41(3):274-9.

9. Morris M, Eifel PJ, Lu J, et al. Pelvic radiation with concurrent chemotherapy compared with pelvic and Para-aortic radiation for high-risk cervical cancer. N Engl J Med. 1999:340:1137-43.

10. Rick LH, Elizabeth $\mathrm{HB}$, Peter WC, et al. Radiation therapy in retroperitoneal sarcoma management. J Surg Oncol. 2018;117:83-93.

11. Porter GA, Baxter NN, Pisters PW. Retroperitoneal sarcoma: a populationbased analysis of epidemiology, surgery, and radiotherapy. Cancer. 2006;106: 1610-6.

12. Baldini EH, Wang $D$, Haas RL, et al. Treatment guidelines for preoperative radiation therapy (RT) for retroperitoneal sarcoma (RPS): preliminary consensus of an international expert panel. Int J Radiat Oncol Biol Phys. 2015;92:602-12

13. Honoré C, Faron M, Mir O, et al. Management of locoregional recurrence after radical resection of a primary nonmetastatic retroperitoneal soft tissue sarcoma: The Gustave Roussy experience. J Surg Oncol. 2018;118(8):1318-25.

14. Garzotto M, Fair WR. Historical perspective on prostate brachytherapy. J Endourol. 2000:14:315-8

15. Davis BJ, Horwitz EM, Lee WR, et al. American brachytherapy society consensus guidelines for transrectal ultrasound-guided permanent prostate brachytherapy. Brachytherapy. 2012;11(1):6-19
16. Martinez AA, Gustafson G, Gonzalez J, et al. Dose escalation using conformal high-dose-rate brachytherapy improves outcome in unfavorable prostate cancer. Int J Radiat Oncol Biol Phys. 2002;53(2):316-27.

17. Wang J, Chai S, Zheng G, et al. Expert consensus statement on computed tomography-guided (125)| radioactive seeds permanent interstitial brachytherapy. J Cancer Res Ther. 2018;14(1):12-7.

18. Nath $\mathrm{R}$, et al. Dosimetry of interstitial brachytherapy sources: recommendations of the AAPM radiation therapy committee task group no. 43. American Association of Physicists in Medicine. Med Phys. 1995;22:20934

19. Rivard MJ, et al. Update of AAPM task group no. 43 report: a revised AAPM protocol for brachytherapy dose calculations. Med Phys. 2004;31:633-74.

20. Eisenhauer EA, Therasse $P$, Bogaerts J, et al. New response evaluation criteria in solid tumours: revised RECIST guideline (version 1.1). Eur J Cancer. 2009; 45(2):228-47. https://doi.org/10.1016/i.ejca.2008.10.026.

21. Common terminology criteria for adverse events (CTCAE) version 5.0, https://evs.nci.nih.gov/ftp1/CTCAE/About.html. Accessed 27 Nov 2017.

22. Hattangadi JA, Powell SN, Macdonald SM, et al. Accelerated partial breast irradiation with low-dose-rate interstitial implant brachytherapy after wide local excision: 12-year outcomes from a prospective trial. [J]. Int J Radiat Oncol Biol Phy. 2011;83(3):791-800.

23. Dalbagni G, Donat SM, Eschwège P, Herr HW, Zelefsky MJ. Results of high dose rate brachytherapy, anterior pelvic exenteration and external beam radiotherapy for carcinoma of the female urethra. J Urol. 2001;166(5):175961.

24. Naghavi AO, Fernandez DC, Mesko N, et al. American brachytherapy society consensus statement for soft tissue sarcoma brachytherapy. Brachytherapy. 2017;16(3):466-89.

25. Jiang YL, Meng N, et al. CT-guided iodine-125 seed permanent implantation for recurrent head and neck cancers. Radiat Oncol. 2010;5:68-75.

26. Huo X, Huo B, Wang H, et al. Percutaneous computed tomography-guided permanent 1251 implantation as therapy for pulmonary metastasis. J Contemp Brachytherapy. 2018;10(2):132-41.

27. Zhang J, Zhang JG, Song T, et al. 1251 seed implant brachytherapy-assisted surgery with preservation of the facial nerve for treatment of malignant parotid gland tumors. Int J Oral Maxillofac Surg. 2008;37:515-20.

28. Mo Z, Zhang T, Zhang Y, et al. Feasibility and clinical value of CT-guided 1251 brachytherapy for metastatic soft tissue sarcoma after first-line chemotherapy failure. Eur Radiol. 2018;28(3):1194-203.

29. Yu LJ, Nan M, Jun JW. Percutaneous computed tomography/ ultrasonography-guided permanent iodine-125 implantation as salvage therapy for recurrent squamous cell cancers of head and neck. Cancer Biol Ther 2010; 9:12, 959-966, DOl: https://doi.org/10.4161/cbt.9.12.11700.

30. Ji Z, Jiang $Y$, Su L, et al. Dosimetry verification of 125-I seeds implantation with three-dimensional printing noncoplanar templates and CT guidance for paravertebral/retroperitoneal malignant tumors. Technol Cancer Res \& Treat. 2017;16(6):1044-50.

31. Yao L, Wang J, Jiang $Y$, et al. Permanent interstitial 125 seed implantation as a salvage therapy for pediatric recurrent or metastatic soft tissue sarcoma after multidisciplinary treatment. World J Surg Oncol. 2015;13(1):335-42.

32. Gross BC, Erkal JL, LOckwood SY, et al. Evaluation of $3 D$ printing and its potential impact on biotechnology and chemical sciences. Anal Chem. 2014;86(7):3240-53.

33. D'Urso PS, Barker TM, Earwaker WJ, et al. Stereolithographic biomodelling in cranio-maxillofacial surgery: a prospective trial. J Craniomaxillofac Surg. 1999;27(1):30-7.

34. Vankoevering KK, Hollister SJ, Green GE. Advances in 3-dimensional printing in otolaryngology. JAMA. 2017;143(2):178-83.

35. Huang MW, Zhang JG, Zheng L, et al. Accuracy evaluation of a 3D-printed individual template for needle guidance in head and neck brachytherapy. J Radiat Res. 2016:57:662-7.

36. Ji Z, Jiang Y, Guo F, et al. Dosimetry verification of radioactive seed implantation for malignant tumors assisted by 3D printing individual templates and CT guidance. Applied Radiat Isotopes. 2017;124:68-74.

\section{Publisher's Note}

Springer Nature remains neutral with regard to jurisdictional claims in published maps and institutional affiliations. 